\section{RISK TO FISH-EATING VERTEBRATES}

Sir, the European Commission Scientific Committee on Health and Environmental Risks (SCHER) has published an updated opinion on the environmental risks and indirect health effects of mercury from dental amalgam at http://ec.europa. eu/health/scientific_committees/ environmental_risks/docs/scher_0_165. pdf. The opinion seeks to cover three areas: Are mercury releases from amalgam a risk to the environment? Does mercury from amalgam which is then released into the environment harm human health? How does environmental risk from mercury in amalgam compare with risks from alternative restorative materials?

The report paints pictures of best to worst case scenarios and concludes that in the local extreme examples of its worst case scenario: maximal dentist density, maximal mercury use and absence of separator devices, the risk of mercury poisoning to fish-eating vertebrates cannot be excluded. Similarly in the extreme worst cases they conclude that mitigation measures might be needed to protect humans from eating contaminated fish. Looking at alternative restorative materials they acknowledge that more information is needed to inform assessments of risk to both environments and to humans. So, until we get a true replacement for amalgam, how as a profession do we respond to this report which acknowledges some risk of harm and some uncertainty?

There are some clinical circumstances where amalgam is still the only appropriate restorative material. If we wish to continue having access to the most appropriate material for those who really need it then we must place ourselves outside the worst case scenario. Amalgam separation is here to stay and we must accept that it is part of the price of using or removing old amalgam. We should also regard amalgam as a restorative material to be avoided unless there is no alternative, yet at the same time we should argue for continued ability to use amalgam when it is the only realistic treatment option. Not perhaps the message we wanted to hear, but a response which gives us a fighting chance of keeping amalgam until a true replacement is developed.

N. Monaghan

Penarth

DOI: 10.1038/sj.bdj.2014.458 to enable best decisions about patient care. However, there has been little discussion in dentistry of the limits of $P$ values in interpreting the results of published studies. This is despite a growing consensus in medicine that the simple use of $P$ values to determine whether the results of a study are valid are insufficient or misleading. ${ }^{1,2}$

Discussion of the limitations of $P$ values are beyond the scope of this letter but there is a growing movement in medicine to include alternative approaches including Bayesian methods. The $P$ value is the probability of observing events as extreme or more extreme than the observed data if the null hypothesis is true. ${ }^{3}$ One of the most common concerns described in the medical literature is that students and clinicians simply end up with an incorrect interpretation of what $\mathrm{P}$ values mean. As described by Goodman, when presenting the results of a study to physicians that the study results had a $P$ value of 0.05 , the majority will state that there is a $95 \%$ or greater chance that the null hypothesis is incorrect. ${ }^{2}$ This is the wrong interpretation because the $P$ value is calculated on the assumption that the null hypothesis is true and it is not a direct measure of the probability that the null hypothesis is false.

Other limitations of the $\mathrm{P}$ value are that it: does not take into consideration the clinical magnitude of the observed association; does not consider biologic plausibility; overstates the evidence against the null hypothesis. ${ }^{4}$ I encourage readers to explore some of the recent published literature in medicine that describe alternative approaches to the analysis of data besides only looking at $P$ values including greater consideration of confidence intervals and the observed clinical magnitude of the associations.

\section{B. Laurence}

Washington DC, USA

1. Goodman S. p values, hypothesis tests, and likelihood: implications for epidemiology of a neglected historical debate. Am J Epidemiol 1993. 137: 485-496.

2. Goodman S. Toward evidence-based medical statistics 1: the P value fallacy. Ann Intern Med 1999; 130: 995-1004.

3. Lee J. Demystify statistical significance - time to move on from the $p$ value to bayesian analysis. J Nat/ Cancer Inst 2011: 103: 2-3.

4. Savitz D. Commentary: reconciling theory and practice: what is to be done with $p$ values? Epidemiology 2013; 24: 212-214.

DOI: $10.1038 /$ sj.bdj.2014.457 